\title{
Crystal structure of the $\mathrm{N}$-terminal domain of VqsR from Pseudomonas aeruginosa at $2.1 \AA$ resolution
}

Received 4 May 2017

Accepted 16 June 2017

Edited by M. S. Weiss, Helmholtz-Zentrum Berlin für Materialien und Energie, Germany

Keywords: VqsR; LuxR; quorum sensing; Pseudomonas aeruginosa.

PDB reference: N-terminal domain of VqsR, $5 \mathrm{xhx}$

Supporting information: this article has supporting information at journals.iucr.org/f

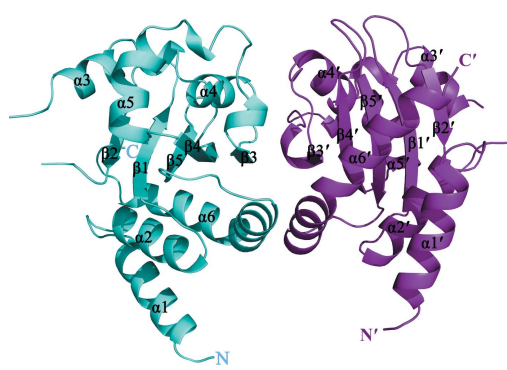

C 2017 International Union of Crystallography

\author{
Qing He, Kang Wang, Tiantian Su, Feng Wang, Lichuan Gu and Sujuan $\mathrm{Xu}^{*}$
}

State Key Laboratory of Microbial Technology, School of Life Sciences, Shandong University, No. 27 Shanda South Road, Jinan, Shandong 250100, People's Republic of China. *Correspondence e-mail: xu_sujuan@sdu.edu.cn

VqsR is a quorum-sensing (QS) transcriptional regulator which controls QS systems (las, $r h l$ and $p q s$ ) by directly downregulating the expression of $q s c R$ in Pseudomonas aeruginosa. As a member of the LuxR family of proteins, VqsR shares the common motif of a helix-turn-helix (HTH)-type DNA-binding domain at the $\mathrm{C}$-terminus, while the function of its $\mathrm{N}$-terminal domain remains obscure. Here, the crystal structure of the N-terminal domain of VqsR (VqsR-N; residues 1-193) was determined at a resolution of $2.1 \AA$. The structure is folded into a regular $\alpha-\beta-\alpha$ sandwich topology, which is similar to the ligand-binding domain (LBD) of the LuxR-type QS receptors. Although their sequence similarity is very low, structural comparison reveals that VqsR-N has a conserved enclosed cavity which could recognize acyl-homoserine lactones (AHLs) as in other LuxR-type AHL receptors. The structure suggests that VqsR could be a potential AHL receptor.

\section{Introduction}

Pseudomonas aeruginosa is a Gram-negative opportunistic human pathogen which causes various types of infections, especially in immunocompromised and cystic fibrosis patients. The pathogenicity of $P$. aeruginosa is mainly owing to the development of biofilms and the expression of extracellular virulence factors (Wu et al., 2000; Hall-Stoodley \& Stoodley, 2005), which are controlled by a cell-density-dependent signalling system termed quorum sensing (QS; Davies et al., 1998; de Kievit \& Iglewski, 2000). Briefly, P. aeruginosa possesses at least three main QS systems: las, rhl and pqs. The two primary QS systems, las and $r h l$, generate the autoinducer signals $N$-3-oxododecanoyl-homoserine lactone $\left(3 \mathrm{O}-\mathrm{C}_{12}\right.$ HSL) and $N$-butanoyl-homoserine lactone ( $\left.\mathrm{C}_{4}-\mathrm{HSL}\right)$, which specifically bind and activate the transcription factors LasR and RhlR, respectively (Bjarnsholt \& Givskov, 2007). The third QS system is the $p q s$ system, which uses alkyl-quinolone signals as autoinducers to activate the LysR-type transcriptional regulator PqsR (Pesci et al., 1999). Each of these systems is modulated at both the transcriptional level and the post-transcriptional level by a group of key regulators, such as Vfr (Albus et al., 1997), RsaL (de Kievit et al., 1999), QteE (Siehnel et al., 2010) and VqsR (Juhas et al., 2004).

VqsR has been characterized as a major virulence and QS regulator in $P$. aeruginosa. Inactivation of the $v q s R$ gene led to decreased acyl-homoserine lactone (AHL) signal production and reduced expression of some QS-controlled virulence factors (Juhas et al., 2004). Gene-chip expression analysis reported that VqsR differentially affected the expression of approximately 200 genes, including numerous genes belonging to the QS network (Juhas et al., 2005). Furthermore, studies revealed that VqsR influences QS systems through directly 
Table 1

Macromolecule-production information.

\begin{tabular}{ll}
\hline Source organism & P. aeruginosa PAO1 \\
DNA source & Genomic DNA \\
Forward primer $\dagger$ & $5^{\prime}$-GAAATTGGATCCATGGATATCGCATTGCACG- \\
& GC-3' \\
Reverse primer $\$$ & $5^{\prime}$-GAAATTCTCGAGTCACTGGACTTCCACCTCC- \\
& GC-3' \\
Cloning vector & pET-15b \\
Expression vector & pET-15b \\
Expression host & E. coli BL21 (DE3) \\
Complete amino-acid sequence & MGSSHHHHHHSGLEVLFQGPGSMDIALHGGAWHE- \\
\multicolumn{1}{c}{ of the construct produced $\$$} & SLGKLLEALDRPFFRILAQTLGQFAPVDNWA- \\
& ALIFSDSSPLILSFMEEEREEVEPDPISRYI- \\
& TGLYLQDPFYQVSRNCRRGGLFHLADIVSEDF- \\
& ETTEYYNTYFAHYVVTDEVQYNVPLGERTLC- \\
& LSLGSESRFGAEQIALFELLRPWVIALMKKRI- \\
& HFEDAVREEAKPIAAAEVEVQ
\end{tabular}

$\dagger$ The BamHI site is underlined. $\ddagger$ The XhoI site is underlined. $\S$ The PreScission Protease recognition sequence is underlined.

controlling the expression of the LasR/RhlR homologue QscR (Liang et al., 2012).

VqsR, encoded by PA2591, is a member of the LuxR family of proteins. Sequence analysis showed that VqsR consists of 268 amino acids, with a helix-turn-helix (HTH)-type DNAbinding domain (residues 198-257) at the C-terminus and a region of unknown function (residues 1-193) at the $\mathrm{N}$-terminus. The structure of VqsR has not been solved to date. In this paper, we determined the structure of the N-terminal domain of VqsR (VqsR-N; residues 1-193) at $2.1 \AA$ resolution. Structure comparison and sequence alignment between VqsR-N and homologous structures revealed that VqsR-N forms a similar $\alpha-\beta-\alpha$ sandwich and has a conserved enclosed cavity, indicating that VqsR could be a potential AHL receptor.

\section{Materials and methods}

\subsection{Macromolecule production}

The coding sequence for VqsR-N (residues 1-193) was amplified by PCR from the $P$. aeruginosa strain PAO1 genome and was inserted into a modified $\mathrm{pET}-15 \mathrm{~b}$ vector with a PreScission Protease cleavage site to remove the His tag. The constructed vector was then transformed into Escherichia coli strain BL21 (DE3) cells. The cells were cultured in LuriaBertani (LB) medium with $100 \mu \mathrm{g} \mathrm{ml}^{-1}$ ampicillin at $310 \mathrm{~K}$ to an $\mathrm{OD}_{600}$ of 0.8 . Expression of VqsR-N was induced with $0.12 \mathrm{~m} M$ isopropyl $\beta$-D-1-thiogalactopyranoside (IPTG) at $289 \mathrm{~K}$ and the induced cells were harvested at $6000 \mathrm{~g}$ for $15 \mathrm{~min}$ at $277 \mathrm{~K}$ after overnight expression.

For protein purification, the cell pellet was resuspended in lysis buffer (25 mM Tris- $\mathrm{HCl} \mathrm{pH} 8.0,200 \mathrm{~m} M \mathrm{NaCl}$ ) and was lysed by sonication. After ultracentrifugation at $28370 \mathrm{~g}$ for $45 \mathrm{~min}$ at $277 \mathrm{~K}$, the supernatant was loaded onto a nickelchelating Sepharose affinity column (GE Healthcare) preequilibrated with lysis buffer. To remove contaminants, the column was washed with wash buffer (25 mM Tris- $\mathrm{HCl}$ pH 8.0, $200 \mathrm{~m} M \mathrm{NaCl}, 10 \mathrm{~m} M$ imidazole), and the Ni-NTA column with His-tagged protein was then incubated with PreScission Protease [100:1(w:w)] at $277 \mathrm{~K}$ overnight to
Table 2

Crystallization.

\begin{tabular}{ll}
\hline Method & Hanging-drop vapour diffusion \\
Temperature (K) & 293 \\
Protein concentration $\left(\mathrm{mg} \mathrm{ml}^{-1}\right)$ & 20 \\
Buffer composition of protein & $10 \mathrm{~m} M$ Tris- $\mathrm{HCl} \mathrm{pH} \mathrm{8.0,100} \mathrm{mM} \mathrm{NaCl}$, \\
$\quad$ solution & $3 \mathrm{~m} M$ DTT \\
Composition of reservoir solution & $0.01 M$ magnesium acetate tetrahydrate, \\
& $0.05 M$ sodium cacodylate trihydrate \\
& $\mathrm{pH} 6.5,1.3 M$ lithium sulfate \\
& monohydrate \\
Volume and ratio of drop & $2 \mu$ l protein solution mixed with $2 \mu \mathrm{l}$ \\
& reservoir solution \\
Volume of reservoir $(\mu \mathrm{l})$ & 500 \\
\hline
\end{tabular}

remove the His tag. The untagged protein was eluted with elution buffer (25 mM Tris- $\mathrm{HCl} \mathrm{pH}$ 8.0, $100 \mathrm{mM} \mathrm{NaCl})$. For further purification, the eluted protein was purified using ionexchange (Source Q, GE Healthcare) and size-exclusion (Superdex 200, GE Healthcare) column chromatography in $10 \mathrm{~m} M$ Tris- $\mathrm{HCl} \mathrm{pH} 8.0,100 \mathrm{~m} M \mathrm{NaCl}, 3 \mathrm{~m} M$ DTT. The purified untagged proteins were collected according to the results of SDS-PAGE, and the final concentration of VqsR-N used for crystallization was approximately $20 \mathrm{mg} \mathrm{ml}^{-1}$. Selenomethionine-labelled (SeMet) VqsR-N was produced by expression from $E$. coli strain BL21 (DE3) harbouring the same pET-15b vector. The bacterial cells were cultured in M9 medium supplemented with specific amino acids as well as selenomethionine as a substitute for methionine (Doublié, 1997). The induction conditions and purification procedure were the same as for the native protein. Macromoleculeproduction information is summarized in Table 1.

\subsection{Crystallization}

Preliminary crystallization experiments were performed using the sitting-drop vapour-diffusion method at 293 K. For initial screening, a 1:1 ratio of protein solution and commercial screen solutions $(1 \mu \mathrm{l}$ of each) was used. After about a week, crystals were observed in several conditions from Crystal Screen. For further optimization, the hanging-drop vapourdiffusion method was used to optimize the crystallization condition by altering the $\mathrm{pH}$ range and salt concentration. Finally, the best crystals were obtained using a reservoir solution consisting of $0.01 M$ magnesium acetate tetrahydrate, $0.05 M$ sodium cacodylate trihydrate $\mathrm{pH}$ 6.5, $1.3 \mathrm{M}$ lithium sulfate monohydrate at $293 \mathrm{~K}$. SeMet VqsR-N crystals were grown using the same solution. Crystallization information is summarized in Table 2.

\subsection{Data collection and processing}

To prevent radiation damage, all crystals were soaked in reservoir buffer containing $15 \%(v / v)$ glycerol and then flashcooled in liquid nitrogen. X-ray diffraction data sets consisting of 180 and 360 images were collected from the native and SeMet crystals, respectively, with an oscillation angle of $1^{\circ}$, an exposure time of $0.5 \mathrm{~s}$ per frame and a crystal-to-detector distance of $250 \mathrm{~mm}$ using a Quantum 315r CCD detector on beamline BL17U1 (wavelength of $0.9791 \AA$ ) at Shanghai 
Table 3

Data collection and processing.

Values in parentheses are for the outer shell.

\begin{tabular}{lll}
\hline & Native VqsR-N & SeMet VqsR-N \\
\hline Diffraction source & BL17U1, SSRF & BL17U1, SSRF \\
Wavelength $(\AA)$ & 0.9791 & 0.9791 \\
Temperature $(\mathrm{K})$ & 100 & 100 \\
Detector & ADSC Quantum & ADSC Quantum \\
& \multicolumn{1}{c}{$315 \mathrm{r}$ CCD } & \multicolumn{1}{c}{$315 \mathrm{r}$ CCD } \\
Crystal-to-detector distance $(\mathrm{mm})$ & 250 & 250 \\
Rotation range per image $\left(^{\circ}\right)$ & 1 & 1 \\
Total rotation range $\left({ }^{\circ}\right)$ & 180 & 360 \\
Exposure time per image $(\mathrm{s})$ & 0.5 & 0.5 \\
Space group & $I 222$ & $I 222$ \\
$a, b, c(\AA)$ & $52.83,135.20$, & $52.98,134.78$, \\
& 150.77 & 150.21 \\
$\alpha, \beta, \gamma\left({ }^{\circ}\right)$ & $90,90,90$ & $90,90,90$ \\
Mosaicity $\left({ }^{\circ}\right)$ & $0.17-0.25$ & $0.26-0.38$ \\
Resolution range $(\AA)$ & $50.00-2.10$ & $50.00-2.20$ \\
& $(2.16-2.10)$ & $(2.28-2.20)$ \\
Total No. of reflections & 226958 & 400208 \\
No. of unique reflections & 32086 & 27810 \\
Completeness $(\%)$ & $99.9(100.0)$ & $100.0(100.0)$ \\
$M u l t i p l i c i t y$ & $7.1(7.4)$ & $14.4(14.9)$ \\
$\langle I / \sigma(I)\rangle$ & $26.04(7.33)$ & $56.93(8.46)$ \\
$R_{\text {r.i.m. }}(\%)$ & $7.3(37.5)$ & $9.7(49.1)$ \\
Overall $B$ factor from Wilson plot $\left(\AA^{2}\right)$ & 34.69 & 37.79 \\
\hline & &
\end{tabular}

Table 4

Structure refinement.

Values in parentheses are for the outer shell.

\begin{tabular}{ll}
\hline Resolution range $(\AA)$ & $36.41-2.10(2.16-2.10)$ \\
Completeness $(\%)$ & 99.8 \\
No. of reflections, working set & 32079 \\
No. of reflections, test set & 1626 \\
Final $R_{\text {cryst }}$ & $0.203(0.217)$ \\
Final $R_{\text {free }}$ & $0.240(0.259)$ \\
No. of non-H atoms & \\
$\quad$ Protein & 2718 \\
$\quad$ Water & 281 \\
Total & 2999 \\
R.m.s. deviations & 0.008 \\
$\quad$ Bonds $(\AA)$ & 0.888 \\
$\quad$ Angles $\left({ }^{\circ}\right)$ & 38.7 \\
Average $B$ factors $\left(\AA^{2}\right)$ & 47.0 \\
$\quad$ Protein & \\
$\quad$ Water & 94.4 \\
Ramachandran plot & 4.6 \\
$\quad$ Favoured regions $(\%)$ & 0.6 \\
$\quad$ Additionally allowed $(\%)$ & \\
$\quad$ Outliers $(\%)$ &
\end{tabular}

Synchrotron Radiation Facility (SSRF), Shanghai, People's Republic of China. Indexing, integration and scaling of the diffraction data were performed with $H K L-2000$ (Otwinowski \& Minor, 1997). Data-collection and processing statistics are given in Table 3.

\subsection{Structure solution and refinement}

The selenium single-wavelength anomalous dispersion (SeSAD) phasing method was used to determine the phase of VqsR-N. Experimental phases were calculated in PHENIX with three heavy atoms identified by AutoSol (Terwilliger et al., 2009). The quality of the electron-density map obtained after solvent flattering enabled the autobuilding of an initial model using AutoBuild in PHENIX (Adams et al., 2010). The initial model was used as a search model for molecular replacement against the native data set using MOLREP in CCP4 (Winn et al., 2011). The resulting model was further refined by alternating rounds of refinement and manual rebuilding using phenix.refine (Afonine et al., 2012) and Coot (Emsley et al., 2010). The $R_{\text {free }}$ value was calculated using 5\% of reflections that were randomly excluded from refinement. The resolution limit of the native data was selected as $2.05 \AA$ based on the $R$ factors, but the structure refinement yielded a high $R_{\text {free }}$ value and a poor Ramachandran plot. The final structure was therefore refined to $2.1 \AA$ resolution. The phasing and final refinement statistics are shown in Table 4. The final model was deposited in the Protein Data Bank (http://www.rcsb.org) as PDB entry 5xhx. All molecular structures in the figures were rendered by PyMOL (http:// www.pymol.org).

\section{Results and discussion \\ 3.1. Overall and subunit structure}

VqsR-N (residues 1-193), which excludes the C-terminal HTH motif, was expressed, purified and crystallized. The crystal structure of VqsR-N belonged to space group I222, with unit-cell parameters $a=52.8, b=135.2, c=150.8 \AA$, and was determined at $2.1 \AA$ resolution. In the refined model, each asymmetric unit contains two monomers, and a comparison of the monomers yields an r.m.s.d. value of $0.8 \AA$, indicating that the two monomers are nearly identical. No density is observed for residues $1-6,63-66$ and $179-193$ of chain $A$, as well as residues 1-6, 63-68 and 176-193 of chain $B$. Although mass spectrometry showed that Cys 94 was not modified in solution, the electron density for Cys 94 indicates that it may be oxidized to a cysteine sulfenic acid (Cys-SOH) in both monomers in the crystal. Similar to other LuxR-type proteins reported in the PDB, VqsR-N forms an $\alpha-\beta-\alpha$ sandwich, which consists of a

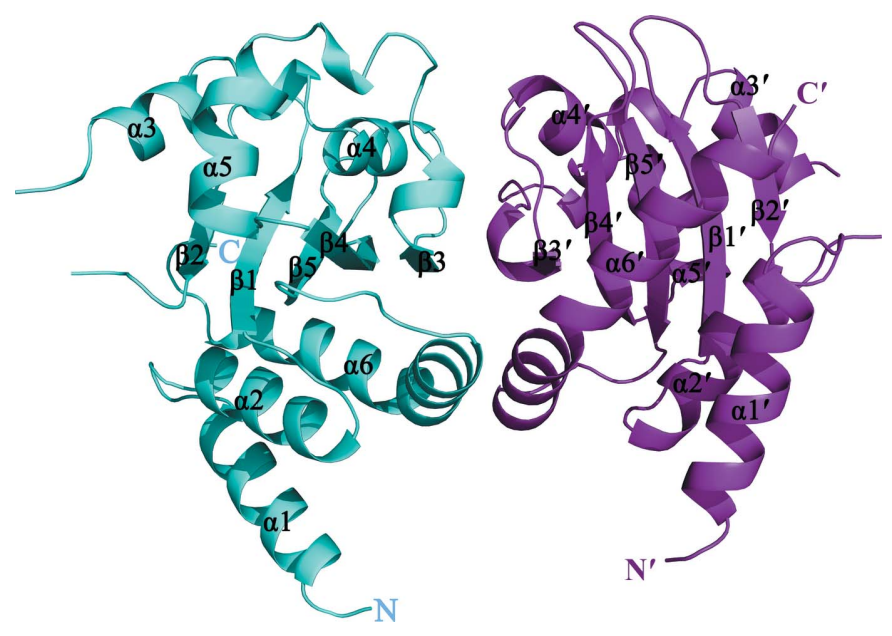

Figure 1

Overall structure of VqsR-N. The structure is depicted as a cartoon diagram. The monomers of this crystallographic dimer are shown in cyan and purple. Secondary-structure elements referred to in the text are labelled. 
five-stranded antiparallel $\beta$-sheet with strand order $2-1-5-4-3$ packed against three $\alpha$-helices on each side (Fig. 1). Three $\alpha$-helices, $\alpha 1$ (residues 9-21), $\alpha 2$ (residues 26-37) and $\alpha 6$ (residues 151-174), form one outer layer of the structure and another three $\alpha$-helices, $\alpha 3$ (residues 70-77), $\alpha 4$ (residues 84 91) and $\alpha 5$ (residues 112-122), form the opposite outer layer. Furthermore, each subunit structure also contains three $3_{10}$-helices. Two subunits form a homodimer mainly through the bending part of the long helix $\alpha 6$ in the crystal.

\subsection{The homodimer structure of VqsR}

VqsR exists as a homodimer in solution, based on gelfiltration results (Liang et al., 2012). As shown in Fig. 1, one asymmetric unit contains two molecules, which dimerize mostly via the short bending part of helix $\alpha 6$ (residues 151-161; Fig. 2a). According to a review of the structures of
LuxR-type homologues, there are several different dimerization forms for the QS receptors, which have differences in the dimeric interface of the ligand-binding domains (LBDs; Kim et al., 2014). We also noticed that the molecules from two neighbouring asymmetric units simultaneously form another dimeric interaction. In this model, helix $\alpha 1$ and most of $\alpha 6$ (residues 159-170) of one subunit interact with most of helix $\alpha 6^{\prime}$ (residues 159-170) and helix $\alpha 1^{\prime}$, respectively, of the other subunit in the dimeric interface (Fig. $2 b$ ). The crystal-packing interactions of both models were analysed using the PISA server (Krissinel \& Henrick, 2007). In the first dimeric model, the area of the interface is $715.0 \AA^{2}$ and the free energy of dissociation is $-7.8 \mathrm{kcal} \mathrm{mol}^{-1}$. For the second model, the values are $938.2 \AA^{2}$ and $-13.6 \mathrm{kcal} \mathrm{mol}^{-1}$, respectively. The larger buried surface area and the smaller free energy of dissociation show that the second model is stable in solution. Furthermore, full-length structures of VqsR were generated

\section{The first model}

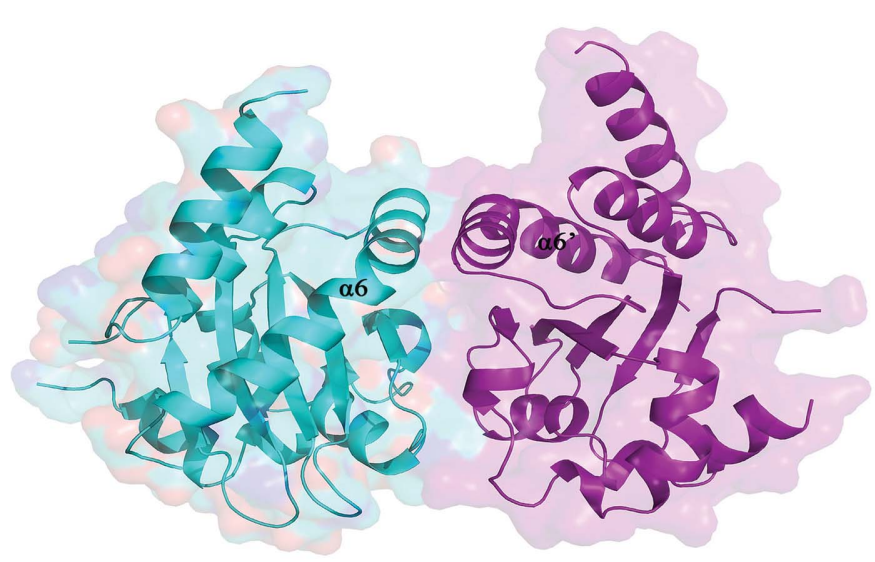

(a)

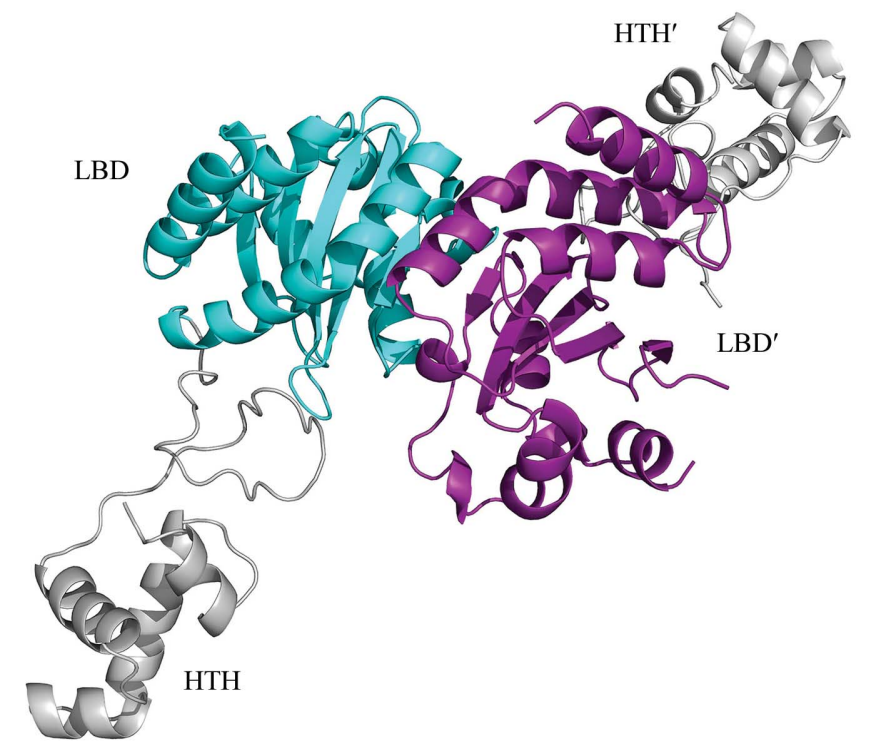

(c)

\section{The second model}

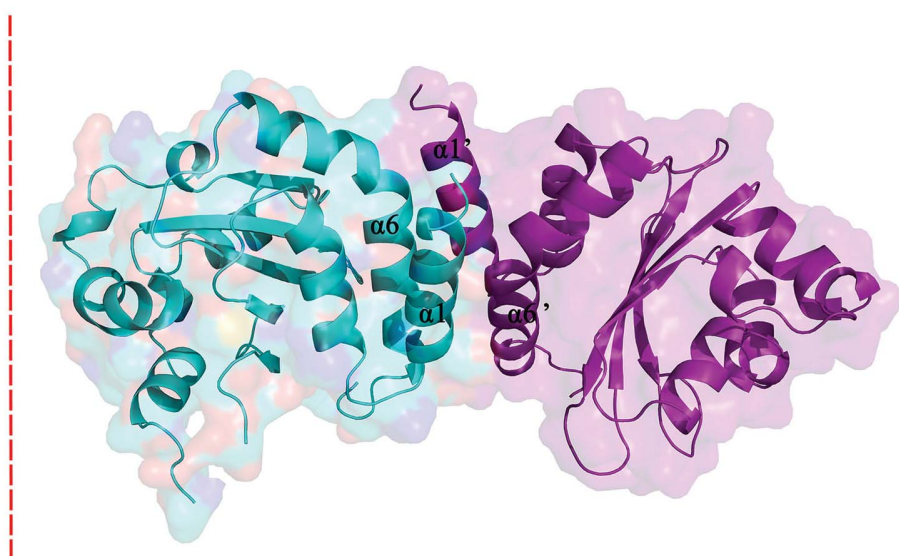

(b)

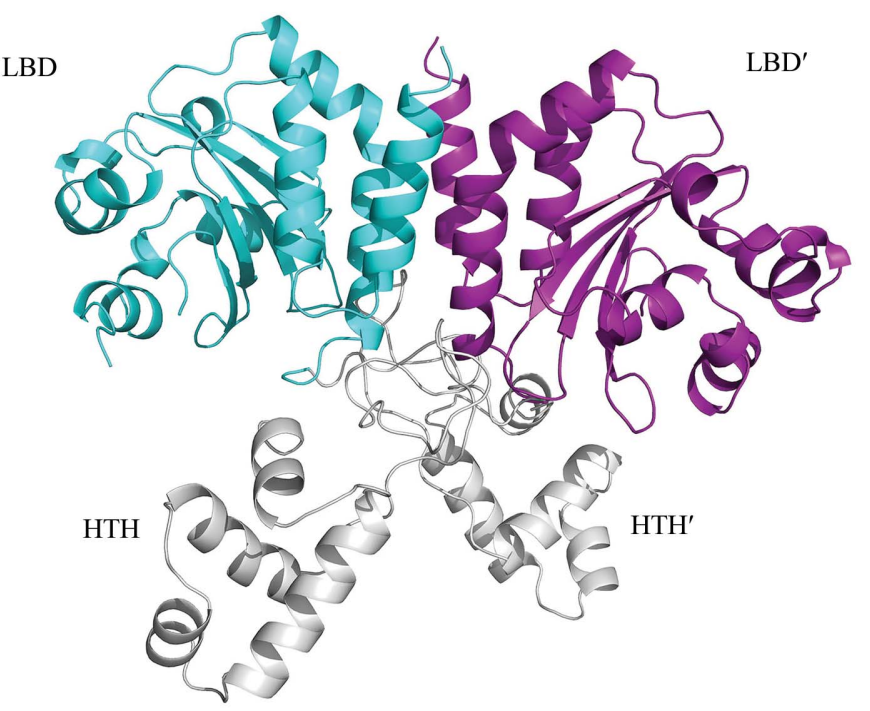

(d)

Figure 2

Homodimer structure of VqsR. $(a, b)$ Cartoon and semi-transparent surface representation of two dimeric models of VqsR-N. The helices involved in the dimeric interface are labelled. $(c, d)$ Cartoon model of the full-length VqsR structure. The two models of the VqsR-N structure are the same as in $(a)$ and (b), while the modelled HTH domains are coloured light grey. 
based on the two dimeric models of VqsR-N (Figs. $2 c$ and $2 d$ ). The HTH domain of VqsR was constructed with the SWISSMODEL web server (Biasini et al., 2014), using the structure of a homologue (PDB code 3qp5; Chen et al., 2011) as a template. The orientations of the two separate HTH domains also indicate that the second dimeric model is more reasonable.

\subsection{Structural comparison between VqsR-N and its} homologues

Despite a lack of sequence homology to any previously determined structures, a $D A L I$ search for globally similar proteins revealed that the overall structure of VqsR-N exhibits an unexpected similar fold to the LBDs of LuxR-type AHL receptors (Holm \& Rosenström, 2010), including TraR (PDB entry 2q0o; Chen et al., 2007), LasR (PDB entry 4ng2; Fan et al., 2013), CviR (PDB entry 3qp5; Chen et al., 2011) and SdiA (PDB entry 4lfu; Kim et al., 2014) (Fig. 3a). The highest $Z$-score for the VqsR-N homologue LasR is 13.6 (r.m.s. deviation of $3.0 \AA$ for 166 equivalent $C^{\alpha}$ positions and $9 \%$ sequence identity). AHLs with various acyl-chain lengths are located in the enclosed cavities of these receptors. Similar to these AHL receptors, VqsR-N also has an enclosed cavity between $\alpha$-helices $\alpha 3-\alpha 5$ and $\beta$-strands $\beta 1-\beta 5$ (Fig. $3 a$ ).

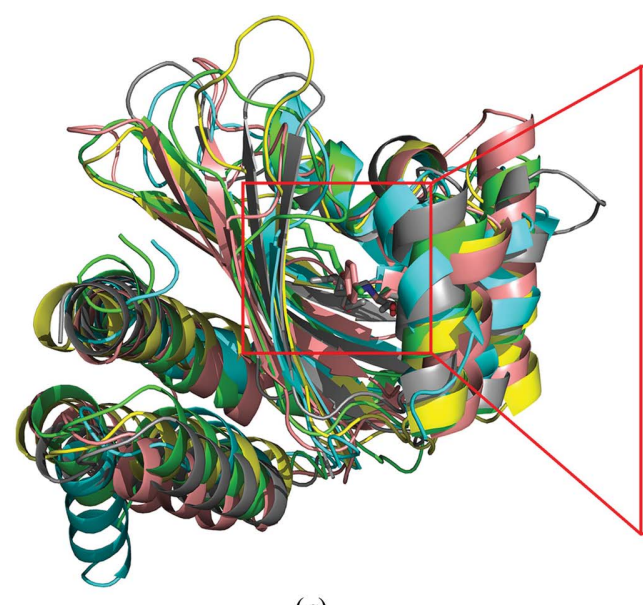

(a)

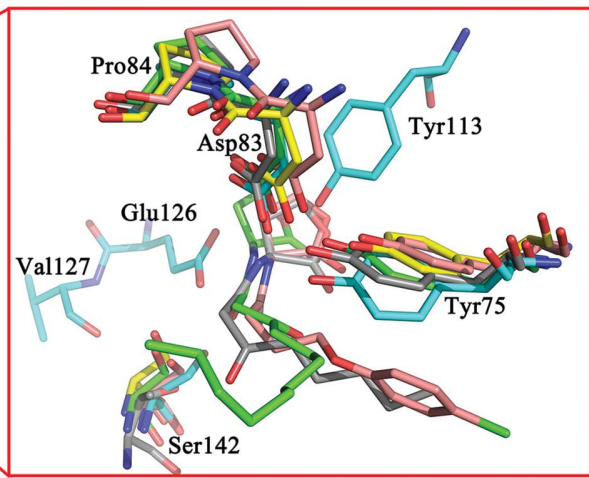

(b)

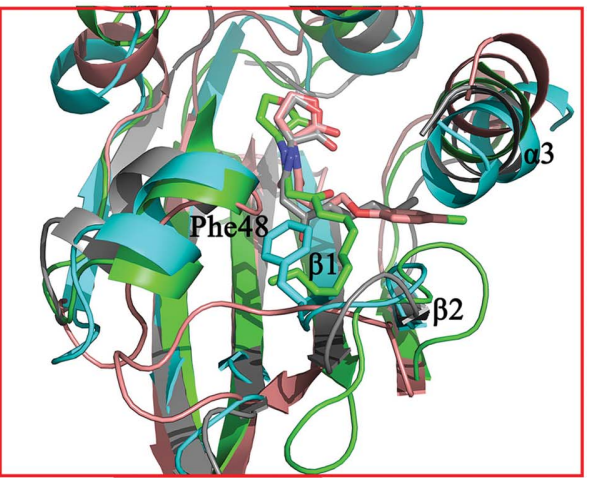

(c) $\alpha 1$

$V q s R \_5 X H X$

VqsR_5XHX

LasR_4NG2

TraR_2Q0O

SidA_4LFU

CviR_3QP5 l. eleeleceleee $\alpha 2$

l... elebeleele $1 \quad 10 \quad 20$

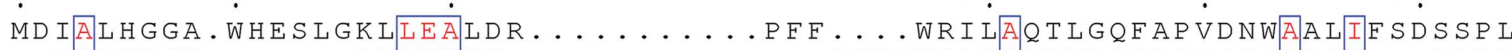

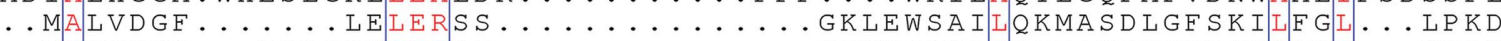

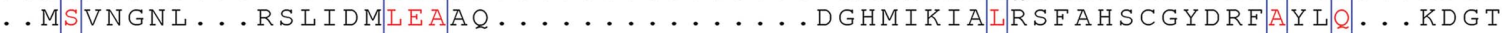
... MQDKDFF SWRRTMLLRFQRME . . . . . . . . . . TAEEVYHEIELQAQQLEYDYYSLCV . . RHPV

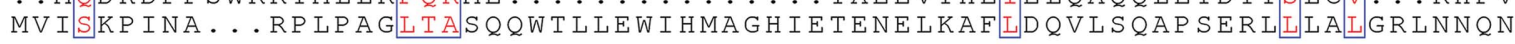

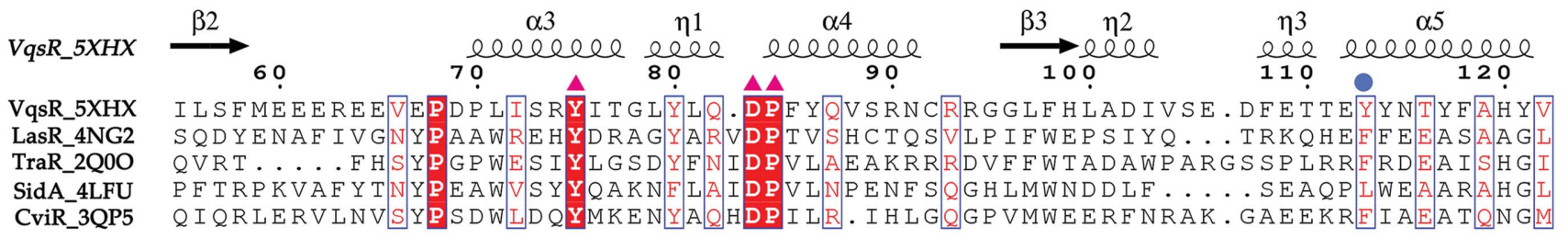

$V q s R \_5 X H X$

VqsR_5XHX LasR_4NG2 TraR_2Q0O SidA_4LFU CviR_3QP5

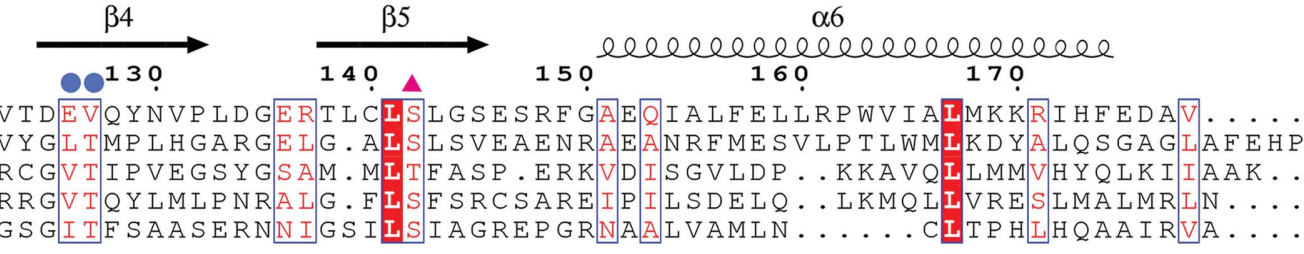

(d)

Figure 3

Structural comparison of VqsR-N with its homologues. (a) VqsR-N (cyan) was superposed with LasR (green), TraR (grey), CviR (salmon) and SdiA (yellow). (b) Residues involved in interaction with the HSL moiety of the AHL are shown as stick models. (c) Close-up view of the AHL-binding cavities of various AHLs in LasR, CviR and TraR. AHLs are shown as stick models in the same colours as in $(a)$. Secondary-structure elements and residues referred to in the text are labelled. (d) Multiple sequence alignment of VqsR-N and its homologues, including LasR, TraR, CviR and SdiA. Conserved residues are shown in blue boxes. The multiple sequence alignment was performed using ESPript (Robert \& Gouet, 2014). Highly conserved residues are shown in white on a red background. Identical and conserved residues stabilizing the HSL moiety of the AHLs are denoted by red triangles and blue dots, respectively. 
Structural comparison showed that VqsR-N possesses several strictly conserved residues which stabilize the homoserine lactone (HSL) head groups of the AHLs in other receptors (Zou \& Nair, 2009), including Tyr75, Asp83, Pro84 and Ser142 (with Thr replacing Ser in TraR). Although Tyr113, Glu126 and Val127 of VqsR-N are not conserved, their equivalents in AHL receptors are also involved in HSL moiety binding, suggesting that the AHLs could be potential signalling molecules for VqsR (Figs. $3 b$ and $3 d$ ). In the AHL-binding cavities, the orientations of $3 \mathrm{O}-\mathrm{C}_{12}$-HSL and AHLs with shorter acyl chains are different (Fig. 3c). Fig. 3(c) shows that the end of the antiparallel $\beta$-strand $\beta 1$ of VqsR-N, especially Phe 48 , makes a steric clash with the acyl chain of $3 \mathrm{O}-\mathrm{C}_{12}$-HSL in the LasR structure. This finding is consistent with a report that VqsR does not bind to 3O-C $\mathrm{C}_{12}$-HSL (Liang et al., 2012). In contrast, AHLs with shorter acyl chains from the CviR and TraR structures can extend into the solvent region between $\alpha$-helix $\alpha 3$ and $\beta$-strand $\beta 2$ of VqsR-N. Our structural analyses suggest that VqsR may be a potential receptor for AHLs with shorter acyl chains.

\section{Acknowledgements}

We are grateful to the staff of beamline BL17U1 at SSRL for support during data collection.

\section{Funding information}

Funding for this research was provided by: The National Basic Research Program of China (973 Program) (award No. 2015 CB150600 to LG).

\section{References}

Adams, P. D. et al. (2010). Acta Cryst. D66, 213-221.

Afonine, P. V., Grosse-Kunstleve, R. W., Echols, N., Headd, J. J., Moriarty, N. W., Mustyakimov, M., Terwilliger, T. C., Urzhumtsev, A., Zwart, P. H. \& Adams, P. D. (2012). Acta Cryst. D68, 352-367.

Albus, A. M., Pesci, E. C., Runyen-Janecky, L. J., West, S. E. \& Iglewski, B. H. (1997). J. Bacteriol. 179, 3928-3935.

Biasini, M., Bienert, S., Waterhouse, A., Arnold, K., Studer, G., Schmidt, T., Kiefer, F., Gallo Cassarino, T., Bertoni, M., Bordoli, L. \& Schwede, T. (2014). Nucleic Acids Res. 42, W252-W258.
Bjarnsholt, T. \& Givskov, M. (2007). Anal. Bioanal. Chem. 387, 409-414.

Chen, G., Jeffrey, P. D., Fuqua, C., Shi, Y. \& Chen, L. (2007). Proc. Natl Acad. Sci. USA, 104, 16474-16479.

Chen, G., Swem, L. R., Swem, D. L., Stauff, D. L., O'Loughlin, C. T., Jeffrey, P. D., Bassler, B. L. \& Hughson, F. M. (2011). Mol. Cell, 42, 199-209.

Davies, D. G., Parsek, M. R., Pearson, J. P., Iglewski, B. H., Costerton, J. W. \& Greenberg, E. P. (1998). Science, 280, 295-298.

Doublié, S. (1997). Methods Enzymol. 276, 523-530.

Emsley, P., Lohkamp, B., Scott, W. G. \& Cowtan, K. (2010). Acta Cryst. D66, 486-501.

Fan, H., Dong, Y., Wu, D., Bowler, M. W., Zhang, L. \& Song, H. (2013). Proc. Natl Acad. Sci. USA, 110, 20765-20770.

Hall-Stoodley, L. \& Stoodley, P. (2005). Trends Microbiol. 13, 7-10.

Holm, L. \& Rosenström, P. (2010). Nucleic Acids Res. 38, W545W549.

Juhas, M., Wiehlmann, L., Huber, B., Jordan, D., Lauber, J., Salunkhe, P., Limpert, A. S., von Götz, F., Steinmetz, I., Eberl, L. \& Tümmler, B. (2004). Microbiology, 150, 831-841.

Juhas, M., Wiehlmann, L., Salunkhe, P., Lauber, J., Buer, J. \& Tümmler, B. (2005). FEMS Microbiol. Lett. 242, 287-295.

Kievit, T. R. de \& Iglewski, B. H. (2000). Infect. Immun. 68, 48394849.

Kievit, T. de, Seed, P. C., Nezezon, J., Passador, L. \& Iglewski, B. H. (1999). J. Bacteriol. 181, 2175-2184.

Kim, T., Duong, T., Wu, C., Choi, J., Lan, N., Kang, S. W., Lokanath, N. K., Shin, D., Hwang, H.-Y. \& Kim, K. K. (2014). Acta Cryst. D70, 694-707.

Krissinel, E. \& Henrick, K. (2007). J. Mol. Biol. 372, 774-797.

Liang, H., Deng, X., Ji, Q., Sun, F., Shen, T. \& He, C. (2012). J. Bacteriol. 194, 3098-3108.

Otwinowski, Z. \& Minor, W. (1997). Methods Enzymol. 276, 307-326.

Pesci, E. C., Milbank, J. B., Pearson, J. P., McKnight, S., Kende, A. S., Greenberg, E. P. \& Iglewski, B. H. (1999). Proc. Natl Acad. Sci. USA, 96, 11229-11234.

Robert, X. \& Gouet, P. (2014). Nucleic Acids Res. 42, W320W324.

Siehnel, R., Traxler, B., An, D. D., Parsek, M. R., Schaefer, A. L. \& Singh, P. K. (2010). Proc. Natl Acad. Sci. USA, 107, 7916-7921.

Terwilliger, T. C., Adams, P. D., Read, R. J., McCoy, A. J., Moriarty, N. W., Grosse-Kunstleve, R. W., Afonine, P. V., Zwart, P. H. \& Hung, L.-W. (2009). Acta Cryst. D65, 582-601.

Winn, M. D. et al. (2011). Acta Cryst. D67, 235-242.

Wu, H., Song, Z., Hentzer, M., Andersen, J. B., Heydorn, A., Mathee, K., Moser, C., Eberl, L., Molin, S., Høiby, N. \& Givskov, M. (2000). Microbiology, 146, 2481-2493.

Zou, Y. \& Nair, S. K. (2009). Chem. Biol. 16, 961-970. 